\title{
The role of right prefrontal and medial cortex in response inhibition: interfering with action restraint and action cancellation using transcranial magnetic brain stimulation
}

Citation for published version (APA):

Dambacher, F., Sack, A. T., Lobbestael, J., Arntz, A., Brugmann, S., \& Schuhmann, T. (2014). The role of right prefrontal and medial cortex in response inhibition: interfering with action restraint and action cancellation using transcranial magnetic brain stimulation. Journal of Cognitive Neuroscience, 26(8), 1775-1784. https://doi.org/10.1162/jocn_a_00595

Document status and date:

Published: 01/01/2014

DOI:

10.1162/jocn_a_00595

Document Version:

Publisher's PDF, also known as Version of record

Document license:

Taverne

Please check the document version of this publication:

- A submitted manuscript is the version of the article upon submission and before peer-review. There can be important differences between the submitted version and the official published version of record. People interested in the research are advised to contact the author for the final version of the publication, or visit the DOI to the publisher's website.

- The final author version and the galley proof are versions of the publication after peer review.

- The final published version features the final layout of the paper including the volume, issue and page numbers.

Link to publication

\footnotetext{
General rights rights.

- You may freely distribute the URL identifying the publication in the public portal. please follow below link for the End User Agreement:

www.umlib.nl/taverne-license

Take down policy

If you believe that this document breaches copyright please contact us at:

repository@maastrichtuniversity.nl

providing details and we will investigate your claim.
}

Copyright and moral rights for the publications made accessible in the public portal are retained by the authors and/or other copyright owners and it is a condition of accessing publications that users recognise and abide by the legal requirements associated with these

- Users may download and print one copy of any publication from the public portal for the purpose of private study or research.

- You may not further distribute the material or use it for any profit-making activity or commercial gain

If the publication is distributed under the terms of Article 25fa of the Dutch Copyright Act, indicated by the "Taverne" license above, 


\title{
The Role of Right Prefrontal and Medial Cortex in Response Inhibition: Interfering with Action Restraint and Action Cancellation Using Transcranial Magnetic Brain Stimulation
}

\author{
Franziska Dambacher, Alexander T. Sack, Jill Lobbestael, Arnoud Arntz, \\ Suzanne Brugmann, and Teresa Schuhmann
}

\begin{abstract}
The ability of inhibiting impulsive urges is paramount for human behavior. Such successful response inhibition has consistently been associated with activity in pFC. The current study aims to unravel the differential involvement of different areas within right $\mathrm{pFC}$ for successful action restraint versus action cancellation. These two conceptually different aspects of action inhibition were measured with a go/no-go task (action restraint) and a stop signal task (action cancellation). Localization of relevant prefrontal activation was based on fMRI data. Significant task-related activation during successful action restraint was localized for each participant individually in right anterior insula (rAI), right superior frontal gyrus, and pre-SMA. Activation during successful action cancellation was localized in rAI, right middle frontal gyrus, and pre-SMA. Subsequently, fMRI-guided continuous
\end{abstract}

\section{INTRODUCTION}

Self-control (or the ability to select, adapt, and withhold behavioral responses) is crucial to human functioning. However, everyday life examples in nonclinical as well as clinical contexts demonstrate how easily the ability to restrain or cancel automatic or preplanned reactions can break down. Neuroscience contributed to this discussion by starting to unravel which neurocorrelates are involved in the failure of inhibitory processing, whereby fMRI has repeatedly emphasized the role of $\mathrm{pFC}$ in controlled behavior and in self-regulation failure (Heatherton \& Wagner, 2011; Miller, 2000). One facet of self-regulation is response (or action) inhibition. Response inhibition, defined as the cognitive ability to withhold any planned or automatic reaction (Logan, Schachar, \& Tannock, 1997), is considered one of the key concepts in understanding the flexible and adaptive nature of human behavior. Neuroimaging studies investigating the involvement of prefrontal neural components in response inhibition mainly used simple motor response inhibition paradigms,

Maastricht University thetaburst stimulation was applied to these regions. Results showed that the disruption of neural activity in rAI reduced both the ability to restrain (go/no-go) and cancel (stop signal) responses. In contrast, continuous thetaburst stimulation-induced disruption of the right superior frontal gyrus specifically impaired the ability to restrain from responding (go/no-go), while leaving the ability for action cancellation largely intact. Stimulation applied to right middle frontal gyrus and pre-SMA did not affect inhibitory processing in neither of the two tasks. These findings provide a more comprehensive perspective on the role of $\mathrm{pFC}$ in inhibition and cognitive control. The results emphasize the role of inferior frontal regions for global inhibition, whereas superior frontal regions seem to be specifically relevant for successful action restraint. focusing on either of two aspects of inhibitory processing: action restraint as measured in go/no-go tasks (GNGTs) and action cancellation as measured in stop signal tasks (SSTs). These imaging studies consistently reported task-related activity within inferior frontal gyrus (IFG) or anterior insula (AI), pre-SMA, and subcortical circuitries involving thalamic regions and the striatum (for reviews, see Swick, Ashley, \& Turken, 2011; Chambers, Garavan, \& Bellgrove, 2009). Within the pFC, mainly dorsolateral pFC, middle frontal gyrus (MFG), and superior frontal gyrus (SFG) have been emphasized (Swick et al., 2011; Simmonds, Pekar, \& Mostofsky, 2008). In most studies, an asymmetric distribution of neural activity toward the right hemisphere was found to play a role in inhibition (Aron, Robbins, \& Poldrack, 2004).

Similarly, the neurocorrelates of response inhibition have also received attention in brain stimulation research. Noninvasive brain stimulation techniques such as transcranial magnetic brain stimulation (TMS) and transcranial direct current stimulation ( $\mathrm{t}$ DCS) can provide further insights into the functional architecture of the response inhibition system in the brain, offering a complimentary methodological perspective to functional imaging approaches 
(Sack \& Linden, 2003). For example, the inhibitory processing in an SST was shown to be impaired by event-related TMS over pre-SMA (Chen, Muggleton, Tzeng, Hung, \& Juan, 2009) and FEF (Muggleton, Chen, Tzeng, Hung, \& Juan, 2010). Repetitive TMS over right IFG (rIFG) compared with a sham TMS condition was shown to reduce inhibitory control in an SST, whereas no effects of stimulation on right MFG (rMFG), right angular gyrus, right and left dorsal premotor cortex, and left IFG were observed (Chambers et al., 2006, 2007). In line with these findings, Verbruggen, Aron, Stevens, and Chambers (2010) showed that the disruption of neural tissue in specifically the ventral part of the rIFG by means of continuous theta burst stimulation (cTBS) impaired response inhibition in a stop signal paradigm. In a tDCS study, Hsu and colleagues (2011) revealed that cathodal stimulation (inhibiting neural activity) over pre-SMA impaired, whereas anodal stimulation (enhancing neural activity) elevated, the ability to successfully inhibit responses in an SST. Jacobson, Javitt, and Lavidor (2011) showed that, whereas cathodal tDCS did not have an effect on response inhibition, anodal tDCS over the rIFG improved inhibitory processing in an SST. Furthermore, effects of cathodal tDCS over right dorsolateral pFC diminishing successful inhibition in a GNGT have been demonstrated (Beeli, Casutt, Baumgartner, \& Jäncke, 2008).

Although converging evidence of these functional brain stimulation studies indicate a causal role of rIFG in successful response inhibition, most of the abovementioned studies focused exclusively on the SST as a measure of action cancellation. Whether or not the same or other neural structures within pFC are relevant for action restraint was not addressed in any of these previous studies. Moreover, although most studies did compare rIFG with other regions within right and left hemisphere, a systematic single subject-based comparison of the differential functional relevance of several distinct subregions within right $\mathrm{pFC}$ for successful action cancellation and/or action restraint is still missing.

The current study was designed to investigate the neural components involved in global response inhibition, as well as action restraint and action cancellation in particular. Virtual lesions were induced in several functional subregions within right $\mathrm{pFC}$ and pre-SMA. cTBS, a repetitive patterned TMS protocol with which longlasting aftereffects can be achieved with relatively short periods of stimulation, was used. Thereby, a 40-sec train including 600 pulses of uninterrupted TBS (three pulses of $50 \mathrm{~Hz}$ repeated every $200 \mathrm{msec}$ ) is delivered (Huang, Edwards, Rounis, Bhatia, \& Rothwell, 2005). The specific target regions were localized for each individual subject based on functional imaging data. The differential effect of brain stimulation on three different prefrontal areas (right AI $[\mathrm{rAI}]$, right SFG [rSFG], rMFG) and pre-SMA was systematically investigated. Individual fMRI-guided neuronavigation ensured that the individual "hotspots" of activations in every single participant were reliably tar- geted across several sessions. Behavioral effects of inducing neural disruptions within the various target regions were examined independently for action restraint (measured with a GNGT) and action cancellation (measured with an SST).

\section{METHODS}

\section{Participants}

All participants $(n=11$; mean age in years $=27, S D=7.27)$ were native Dutch speakers, had normal or corrected-tonormal vision, and had no history of neurological or psychiatric disorders. They received medical approval from an independent physician for participation and gave their written informed consent after being introduced to the procedure. The study was approved by the local medical ethical committee.

\section{Experimental Design}

Participants took part in one training session and five experimental sessions. During the training session, they were introduced to the TMS laboratories and the tasks they had to perform. Furthermore, their individual active motor threshold was determined. In each of the experimental sessions, participants received cTBS (Huang et al., 2005) on one of four target sites or sham stimulation in a semicounterbalanced order. rSFG, rMFG, rAI, and preSMA were identified as target sites based on individual fMRI data (details presented below). Sham TMS was delivered on a target site located in the middle of the four empirical target sites to keep nonneural TMS effects constant (Duecker \& Sack, 2013). Therefore, Talairach coordinates of the four sites were averaged and transferred back to native space to define a sham TMS target site. This procedure ensured sham TMS to be an optimally matched control condition for each specific empirical target site, especially with respect to the mimicking of the auditory sensation accompanying cTBS.

\section{Paradigms}

To access response inhibition, two paradigms were employed: a GNGT and an SST. The task designs were comparable to enable the direct comparison of the outcome measures.

To elicit action restraint, a simple go/no-go motor response task was employed (Figure 1A). Participants were instructed to respond as fast and accurately as possible to a frequent go stimulus via button press with the right index finger, while they should not respond to a rare no-go stimulus. Go as well as no-go stimuli were presented for $100 \mathrm{msec}$. Intertrial intervals were randomly varied among five levels $(650,750,850,950$, or $1050 \mathrm{msec})$ to avoid expectancy effects. 
Figure 1. Task design. Go trials, color-coded in green; no-go/stop trials, color-coded in red. (A) GNGT: Participants were instructed to respond as fast and accurately as possible to a frequent go stimulus (in this case a C) via button press, while they should not respond to a rare no-go stimulus (in this case an M). (B) SST: Participants were instructed to respond as fast and accurately as possible to a go stimulus (in this case a $\mathrm{C}$ ) via button press, while they should not respond to this same stimulus in the rare cases when it was followed by a visual stop signal (in this case an M).

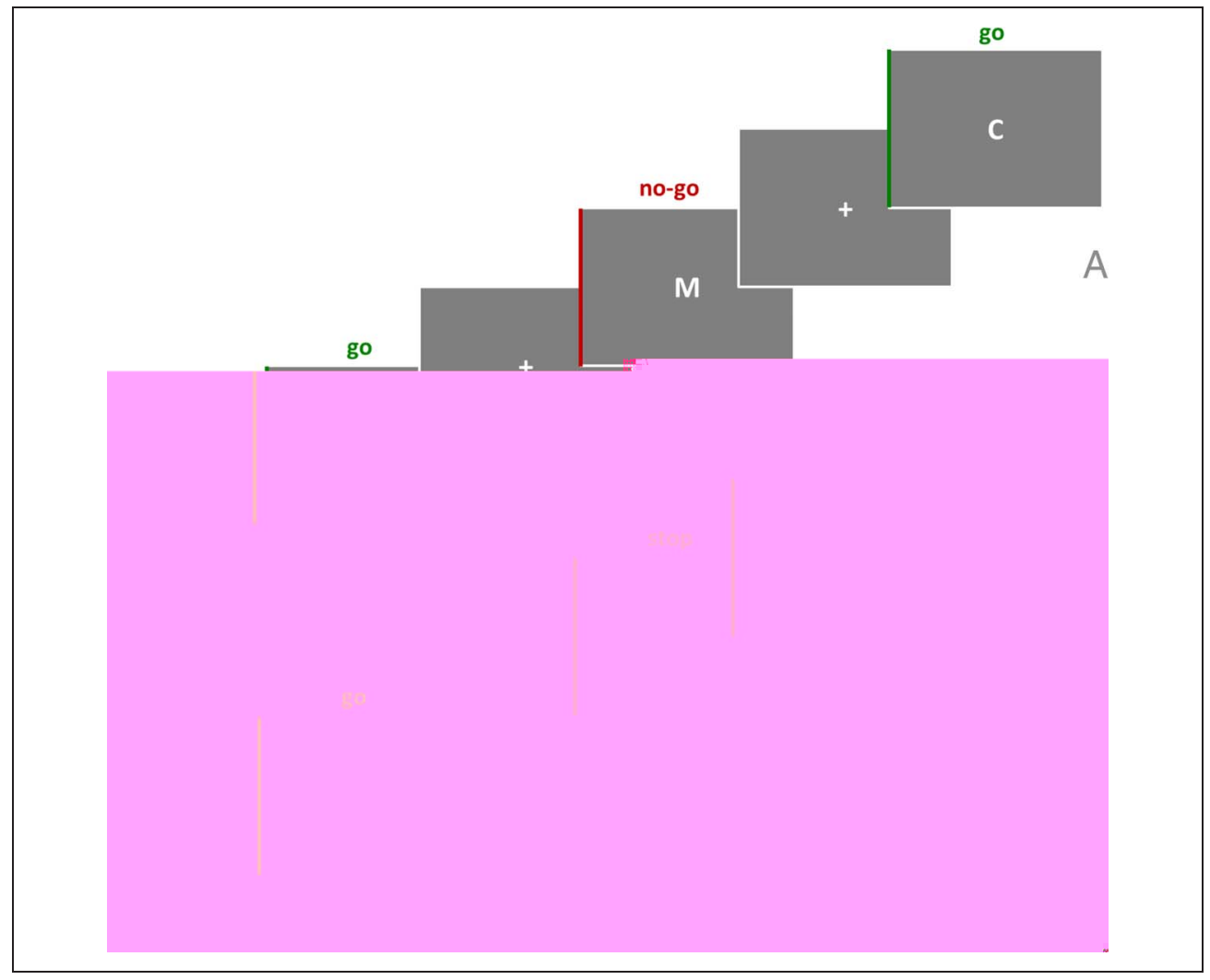

To elicit action cancellation, a modified SST (Logan et al., 1997) was employed (Figure 1B). Participants were instructed to respond as fast and accurately as possible to a go stimulus via button press with the right index finger, while they should not respond to the very same stimulus in the rare cases when it was followed by a visual stop signal. No dual choice RT task was implemented to keep the design of the two tasks as similar as possible. This means that the go stimulus was presented in all trials and the stop stimulus was presented following the go stimulus only during stop trials. In the go trials, the go stimulus was presented for $100 \mathrm{msec}$. In the stop trials the go stimulus was presented for $100 \mathrm{msec}$ followed with a SOA of 150 , $200,250,300$, or $350 \mathrm{msec}$ by a visual stop signal presented for $80 \mathrm{msec}$. Intertrial intervals were jittered (1050 and $1250 \mathrm{msec}$ ) to avoid expectancy effects.

The letters $\mathrm{C}$ and $\mathrm{M}$ were used as stimuli, because they do not have any linguistic association with the concept of "stopping." The assigned letter to the go and no-go/stop condition was randomized between participants. Stimuli and fixation crosses were presented in white (RGB 255/ 255/255; Arial 24 pt.) on a gray background (RGB 125/ $125 / 125)$. For each task, participants had to complete five blocks of 64 trials including 25\% inhibition trials. Go and no-go/stop trials were pseudorandomized (one of four trials was an inhibition trial) to prevent randomizations including trains of more than two consecutive inhibition trials. This design led to a total of 320 trials (80 inhibition trials) per task. After each block, participants received feedback on their mean RTs for go trials, their number of misses in go trials, and their percentage of false alarms in inhibition trials. During the training session, participants were familiarized with the tasks and our procedure and had to complete a training version of each task. Stimuli were presented using Presentation software (Neurobehavioural Systems, Inc., Albany, CA). The order of the two tasks within one session was counterbalanced within and semicounterbalanced between participants.

\section{Localizations of TMS Target Regions}

Previous to their participation in the current experiment, all participants underwent structural (high-resolution T1weighted MP-RAGE, isotropic voxel resolution $1 \times 1 \times$ $1 \mathrm{~mm}^{3}, 192$ sagittal slices) and functional whole-brain (gradient-echo EPI sequence, repetition time $=1500 \mathrm{msec}$, echo time $=28 \mathrm{msec}$, field of view $=224 \mathrm{~mm}$, flip angle $=$ $71^{\circ}$, matrix $=64 \times 64$, slice thickness $=3.5 \mathrm{~mm}$, distance factor $=10 \%, 458$ volumes per run) imaging in a $3 \mathrm{~T}$ Siemens Allegra Scanner. All fMRI analyses and 3-D surface reconstructions were performed with Brain Voyager QX (Brain Innovation BV, Maastricht, Netherlands). For 17 participants, random effects group analyses $(p<.001$, cluster level threshold corrected) of successful inhibition in two fMRI adapted versions of the exact same tasks as described above were conducted. Functional imaging revealed three right-lateralized frontal brain regions as being mainly activated during action restraint and/or action cancellation (rSFG for the GNGT, rMFG for the SST; rAI/IFG and pre-SMA for both tasks; Figure 2). Eleven participants 


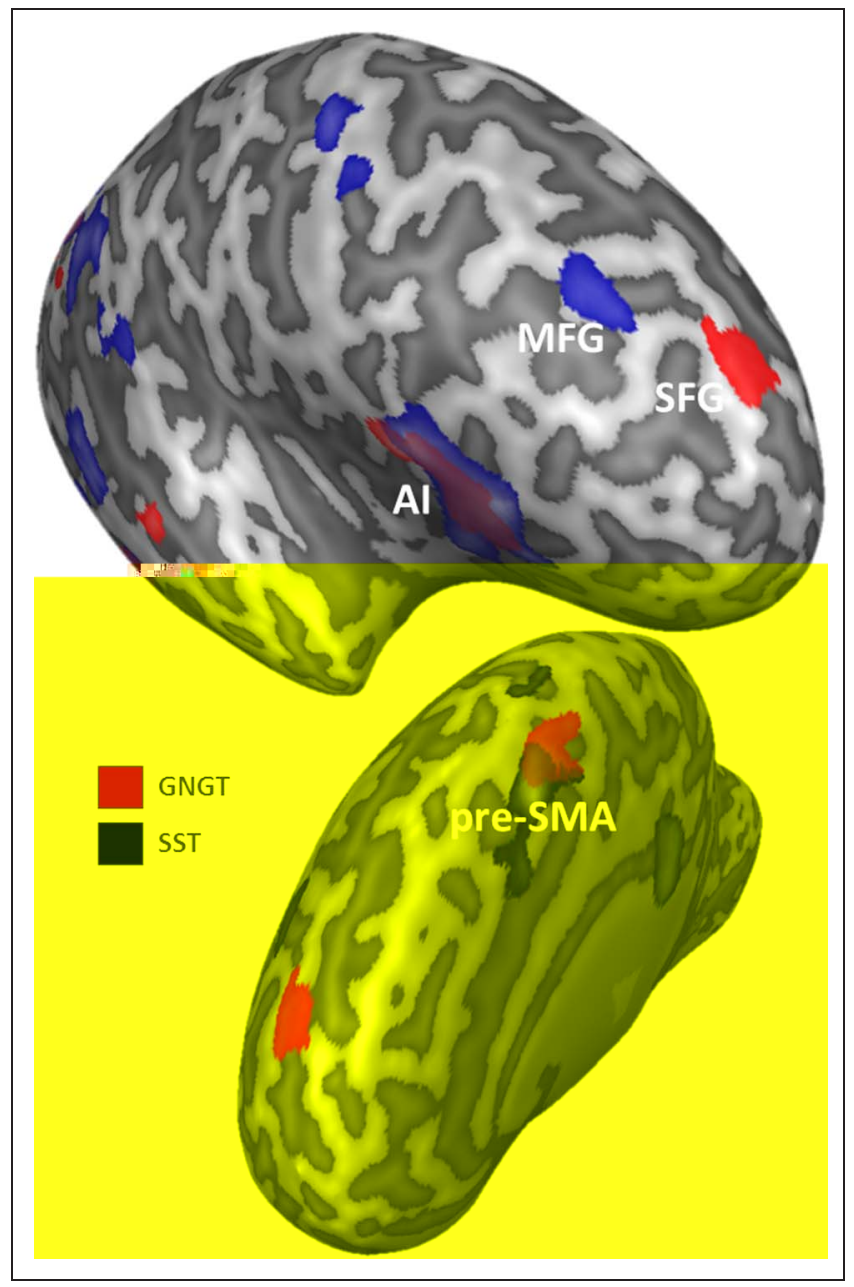

Figure 2. Increased cortical activation associated with successful inhibition during no-go (color-coded in red) and stop (color-coded in blue) trials. Lateral and medial view of an inflated right hemisphere. Statistical maps $p \leq .001 \operatorname{RFX} n=17$.

from the sample were medically eligible to undergo TMS and agreed to come back for further sessions. For them, the described regions were localized on single subject maps choosing the statistically most significant voxels of individual activation within the regions. The established target regions were transformed into surface clusters and projected onto the individual 3-D surface reconstruction of each participant's right hemisphere (Figure 3; for specific individual Talairach coordinates, see Table 1). The average distance from the skull surface to the target site was $50 \mathrm{~mm}$ for rAI, $56 \mathrm{~mm}$ for pre-SMA, $33 \mathrm{~mm}$ for $\mathrm{rMFG}$, and $34 \mathrm{~mm}$ for rSFG.

\section{TMS Stimulation}

Biphasic TMS pulses were applied using the Medtronic MagPro X100 stimulator (Medtronic Functional Diagnostics A/S, Skovlunde, Denmark, maximum stimulator output approximately $1.9 \mathrm{~T}$ and $150 \mathrm{~A} / \mu \mathrm{s}$ ) and a figure-of-eight coil (MCB70; with the inner and outer radii of the two coil loops $=1.2$ and $5.4 \mathrm{~cm}$, respectively). The coil was manually held tangentially to the skull, oriented perpendicularly to the gyrus on which the target site was located. The heads of the participants were coregistered to their structural brain imaging data using the online visualization function of Brain Voyager TMS Neuronavigator (Brain Innovation BV). This allowed targeting brain areas precisely based on functional imaging data projected onto the 3-D surface reconstructions of the participants' right hemispheres and monitoring the coil position while stimulating. CTBS was applied at 100\% individual active motor threshold (ranging from 25\% to 36\% of maximum stimulator output) for $40 \mathrm{sec}$. Active motor threshold was determined by the observation of movement method as proposed by Varnava, Stokes, and Chambers (2011). For sham stimulation, a placebo figure-of-eight TMS coil (MC-PB70 Placebo) was used. In the sham condition, the neuronavigation procedure and all other stimulation parameters were equal to the real TMS conditions.

\section{Statistical Analysis of Behavioral Data}

One participant did not complete one experimental session because of uncomfortable twitches in the face muscles. Therefore, this participant was excluded from the analyses. Ten participants completed all six sessions. Two participants had to be excluded because of behavioral false alarm pattern incompatible with the given task instruction (one participant showed false alarm rates on the GNGT entirely opposite to all other participants with an outlier analysis identifying his differential values as being $>1 S D$ from the mean, and a second participant showed a false alarm rate on inhibition trials in the SST of $100 \%$ at baseline). Therefore, data of eight participants was included in our analysis. Our sample size was evaluated by a power analysis based on the average effect sizes revealed by Verbruggen et al. (2010) and Chambers et al. (2006, 2007) and the ratio to which a functionally guided localization of TMS target regions increases statistical power compared with a conventional approach (Sack et al., 2009). The power analysis was performed using G-power software 3.1.5 (Faul, Erdfelder, Lang, \& Buchner, 2007; assumptions: $\alpha 0.05,1-\beta$ 0.95).

For every participant and both tasks, mean RT, misses in go trials, and false alarms in inhibition trials were calculated. For the SST additionally the mean stop signal RT (SSRT) was estimated according to the horse-race model (Band, Van der Molen, \& Logan, 2003). SSRT was defined per participant as the difference between stop signal delay at 50\% correct inhibition and the median RT. All further statistical analyses were performed using SPSS19 (IBM Statistics, Armonk, NY). To examine statistical differences in performance with respect to the five TMS conditions, a general linear mixed model analysis including the four stimulation sites as dummy coded variables with the sham condition as the reference variable was performed. Thereby, four a priori comparisons (each stimulation 
Figure 3. Individual fMRIbased brain stimulation target sites for eighth participant. Stimulation sites for which an effect of TMS on action cancellation could be demonstrated in prior studies (Verbruggen et al., 2010; Chambers et al., 2006, 2007) are shown color-coded in gray.

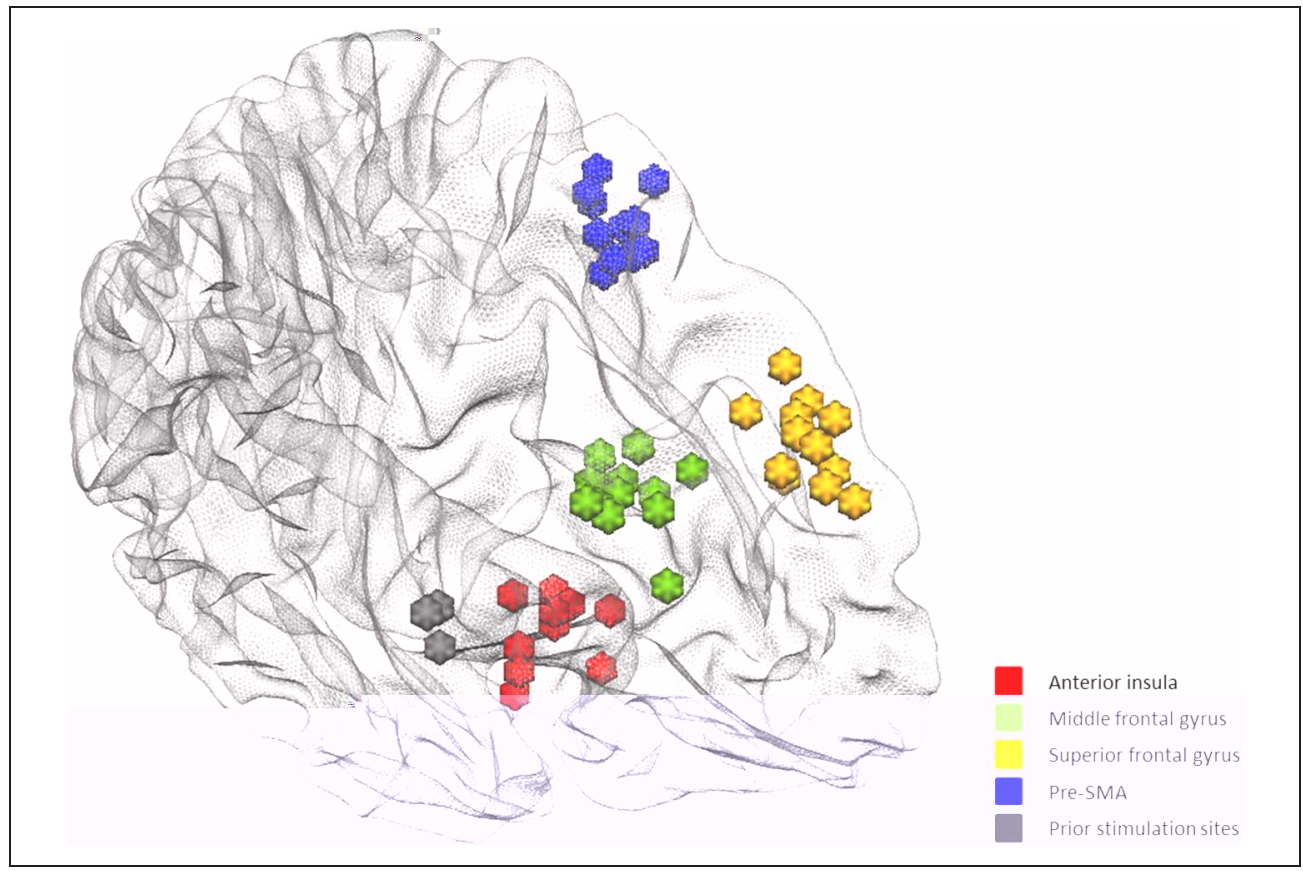

site compared with sham condition) could be tested. Mixed models provide an approach to repeated-measures data in the framework of regression analyses. Order effects with respect to the individual sessions for false alarm rates were tested in a one-factorial ANOVA with repeated measures.

\section{RESULTS}

The presented analyses are based on data of eight participants. In the neutral (sham stimulation) condition, participants reacted faster in the successful go trials for the GNGT compared with the SST (GNGT: $301.06 \pm$ $14.65 \mathrm{msec}$; SST: $455.25 \pm 44.96 \mathrm{msec})$. False alarms (commission errors on inhibition trials) were more frequent in stop trials than in no-go trials (GNGT: $16.00 \pm$ 3.42; SST: $33.63 \pm 5.89$ ). In both tasks, relatively few misses (omission errors on go trials) occurred (GNGT: $5.13 \pm 3.15$; SST: $2.38 \pm 0.82$ ). In the SST, an average SSRT of $166.52 \mathrm{msec}$ was observed ( $S D=102.77)$. However, no perfect positive linear relationship between false alarm rates and length of SSD (more false alarms the longer the SSD) as foreseen based on the simulations by Band et al. (2003) was found in our data. Furthermore,

Table 1. Talairach Coordinates of Individual Brain Stimulation Target Sites for Every Participant

\begin{tabular}{|c|c|c|c|c|c|c|c|c|c|c|c|c|}
\hline \multirow[b]{2}{*}{ Participant } & \multicolumn{3}{|c|}{$r A I$} & \multicolumn{3}{|c|}{ Pre-SMA } & \multicolumn{3}{|c|}{$r M F G$} & \multicolumn{3}{|c|}{$r S F G$} \\
\hline & $x$ & $y$ & $z$ & $x$ & $y$ & $z$ & $x$ & $y$ & $z$ & $x$ & $y$ & $z$ \\
\hline Group $(n=17)$ & 33 & 24 & 9 & 3 & 5 & 49 & 37 & 32 & 34 & 21 & 49 & 43 \\
\hline 1 & 39 & 15 & 4 & 5 & 6 & 50 & 27 & 36 & 35 & 20 & 58 & 36 \\
\hline 2 & 40 & 16 & 1 & 2 & -5 & 58 & 44 & 33 & 36 & 18 & 44 & 50 \\
\hline 3 & 32 & 15 & 10 & 6 & 0 & 45 & 41 & 32 & 34 & 17 & 52 & 37 \\
\hline 4 & 37 & 20 & 10 & 5 & -2 & 50 & 31 & 33 & 32 & 20 & 46 & 35 \\
\hline 5 & 28 & 22 & 8 & 5 & 5 & 54 & 36 & 26 & 33 & 16 & 51 & 44 \\
\hline 6 & 37 & 20 & 11 & 1 & 5 & 59 & 42 & 35 & 34 & 22 & 53 & 43 \\
\hline 7 & 34 & 26 & 3 & 4 & 2 & 52 & 31 & 23 & 34 & 12 & 43 & 42 \\
\hline 8 & 38 & 13 & -5 & 8 & 4 & 49 & 35 & 39 & 22 & 21 & 54 & 37 \\
\hline 9 & 39 & 14 & 12 & 5 & 5 & 49 & 34 & 30 & 34 & 21 & 40 & 43 \\
\hline 10 & 33 & 19 & 10 & 4 & -4 & 54 & 31 & 30 & 38 & 28 & 48 & 38 \\
\hline
\end{tabular}


an erroneous estimation of SSRT might have been caused by the fact that we did not employ a dual choice RT paradigm as is often done in SST research. Therefore, the interpretability of SSRT in our study is questionable. Focusing on the significantly longer RTs in SST compared with GNGT performance, one could assume that participants just waited for the stop signal and thereby transformed the SST simply into a slower GNGT. This is known to distort SSRT estimation (Verbruggen, Chambers, \& Logan, 2013). However, the significantly higher error rate in the SST as well as the fact that we find differential TMS results for both paradigms speaks against this interpretation.

\section{Effects of Stimulation for the GNGT}

For action restraint, the mixed model analysis revealed that compared with sham stimulation false alarm rates in inhibition trials were elevated following rAI stimulation (mean difference compared with sham condition $=6.25$, $F=6.0, d f=32, p=.021$, Cohen's $d=0.59$ ) and rSFG stimulation (mean difference compared with sham condition $=6.38, F=6.24, d f=32, p=.019$, Cohen's $d=$ 0.51). No significant effects on false alarm rates were found for pre-SMA and rMFG stimulation (mean difference pre-SMA compared with sham condition $=2.25$, $F=0.77, d f=32, p=.385$; mean difference $\mathrm{rMFG}$ compared with sham condition $=1.38, F=0.29, d f=32$, $p=.594)$. An ANOVA revealed no order effects with respect to the individual sessions for false alarm rates induced by for instance learning or habituation, $F(4,28)=0.45, p=$ 769. Stimulation did not significantly affect response rates/misses (rAI: $F=0.04, d f=32, p=.843$; pre-SMA: $F=0.42, d f=32, p=.521 ;$ rMFG: $F=0.06, d f=32, p=$ .805 ; rSFG: $F=0.09, d f=32, p=.767$ ) and RTs (rAI: $F=$ $1.44, d f=32, p=.240$; pre-SMA: $F=0.05, d f=32, p=.821$; rMFG: $F=0.20, d f=32, p=.656$; rSFG: $F=2.09, d f=32$, $p=.158)$ in go trials. Significant results are depicted in Figure 4 .

\section{Effects of Stimulation for the SST}

For action cancellation, the mixed model analysis revealed that compared with sham stimulation false alarm rates in inhibition trials were elevated following rAI stimulation (mean difference compared with sham condition = 6.13, $F=4.30, d f=31, p=.048$, Cohen's $d=0.32$ ). No significant effects on false alarm rates were found for pre-SMA, rMFG, and rSFG stimulation (mean difference pre-SMA compared with sham condition $=3.75, F=$ $1.0, d f=31, p=.305$; mean difference rMFG compared with sham condition $=2.63, F=0.31, d f=31, p=.582$; mean difference $\mathrm{rSFG}$ compared with sham condition $=$ $3.50, F=0.67, d f=31, p=.357)$. An ANOVA revealed no order effects with respect to the individual sessions for false alarm rates induced by for instance learning or habituation, $F(4,28)=1.29, p=.297$. Stimulation did

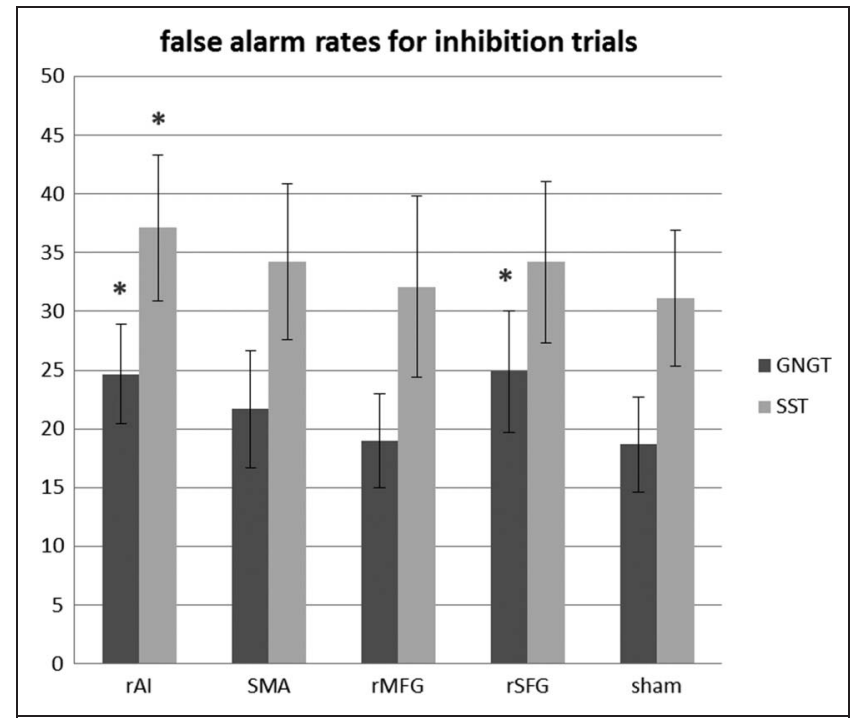

Figure 4. False alarm rates in inhibition trials for action restraint and action cancellation. Significant elevation of false alarm rates compared with the sham condition are labeled $(* p>.05)$. sham $=$ sham brain stimulation.

not significantly affect response rates/misses in go trials (rAI: $F=0.00, d f=31, p=.995$; pre-SMA: $F=0.07, d f=$ $31, p=.786 ;$ rMFG: $F=0.30, d f=31, p=.584$; rSFG: $F=0.48, d f=31, p=.493$ ) and SSRTs (rAI: $F=0.01, d f=$ $31, p=.908$; pre-SMA: $F=0.16, d f=31, p=.690$; rMFG: $F=0.00, d f=31, p=.939 ;$ rSFG $: F=1.23, d f=31, p=$ .277 ). After pre-SMA stimulation participants reacted significantly faster on go trials (mean difference compared with sham condition $=11.63 \mathrm{msec}, F=4.51, d f=31, p=.043$ ). Otherwise, RTs (rAI: $F=3.39, d f=31, p=.076$; rMFG: $F=$ $0.09, d f=31, p=.758 ;$ rSFG: $F=2.43, d f=31, p=.130)$ in go trials have not been affected by stimulation. Significant results are depicted in Figure 4.

\section{DISCUSSION}

This study systematically investigated the role of right pFC in controlled behavior, more specifically in response inhibition. The disruption of neural activity in right inferior frontal regions as well as pre-SMA by means of cTBS was expected to corrupt the ability to generally inhibit responses. Furthermore, the disruption of rSFG activity was expected to impair action restraint (as measured with a GNGT) but not cancellation (as measured with an SST), whereas the disruption of rMFG was thought to impair action cancellation but not restraint. Results showed that inferior frontal regions are crucial for different aspects of inhibitory processing (action restraint and action cancellation) whereas superior frontal regions might be more relevant for action restraint as measured in go/no-go paradigms specifically. Stimulation applied to 
middle frontal regions and pre-SMA did not have any effect on inhibitory processing.

\section{Right Inferior Frontal Cortex}

According to our expectations, the disruption of neural activity in right inferior frontal cortex strongly affected inhibitory processing in general. CTBS to rAI impaired the ability to restrain responses (measured with a GNGT) as well as the ability to cancel reactions to a certain configuration of stimuli (measured with an SST). This is in line with a vast body of imaging literature emphasizing the crucial role of right inferior frontal cortex in inhibition (for a review, see Aron et al., 2004). Right inferior frontal regions have been found to be activated during successful inhibition across different response inhibition modalities. Thereby, paradigms such as the stop signal (Boehler, Appelbaum, Krebs, Hopf, \& Woldorff, 2010; Duann, Ide, Luo, \& Li, 2009; for reviews, see Swick et al., 2011; Chambers et al., 2009), the go/no-go (for reviews, see Swick et al., 2011; Simmonds et al., 2008), and the anti-saccade task (Chikazoe, Konishi, Asari, Jimura, \& Miyashita, 2007) have been employed. Furthermore, all brain stimulation studies on response inhibition that included right inferior frontal regions as target sites demonstrated an effect of brain stimulation on inhibition even across different stimulation modalities (rTMS, Chambers et al., 2006, 2007; cTBS, Verbruggen et al., 2010; tDCS, Jacobson et al., 2011). Thus, our results further support the notion of right inferior frontal cortex being crucial for successful action inhibition and emphasize that this holds true even across different aspects of inhibitory processing employing a within-subject approach. However, there are two issues to consider when interpreting our findings.

So far, within the inferior frontal cortex specifically the IFG has been subject of brain stimulation research. Our study is the first to not target rIFG, but rAI. The decision to target rAI was made with respect to our fMRI findings on which the localization of individual target regions was based. Although with sufficiently liberal significance level extending into rIFG, the most significant "hotspot" of inferior frontal activation during successful inhibition-for both action restraint and action cancellation-was found to be localized within the AI. This was the case for analyses of action restraint and action cancellation independently on group and also single subject level in our data set. In their meta-analysis of 42 studies of GNGTs and SSTs, Swick and colleagues (2011) also found the most prominent overlap of inferior frontal activation to be in AI. Sharp and colleagues (2010) labeled their focus of activation as "IFG/ insula" and thereby acknowledged the role of AI alongside IFG activation in response inhibition research. With respect to cTBS stimulation, however, one has to be aware of the fact that deeper brain structures cannot be targeted without also stimulating the more superficial regions lying on top. Therefore, we have to emphasize that, by targeting their individual "hotspot" of activation during successful inhibi- tion within AI, for some participants the most anterior part of the IFG (around BA13; see Figure 3) was also exposed to stimulation.

Furthermore, labeling the function of inferior frontal regions in response inhibition merely as "inhibition" has to be evaluated with caution. Hampshire, Chamberlain, Monti, Duncan, and Owen (2010) concluded from their imaging study that inferior frontal cortex might play a more attentional than inhibitory role in response inhibition. They argue that IFG is recruited as soon as important cues have to be processed, regardless of whether a motor response has to be generated or inhibited. However, our findings clearly show that performance on trials in which the generation of a motor response is requested (go trials) is not affected by the disruption of neural activity in inferior frontal regions whereas the successful inhibition of a motor response (in no-go and stop trials) is. Verbruggen and colleagues (2010) further specified the effect on response inhibition. They showed that the disruption of neural tissue in specifically the ventral part of the rIFG by means of cTBS did affect both stop signal trials (in which subjects had to withhold their response) and dual signal trials (in which subjects had to execute an additional response). The more dorsal part of the rIFG seemed more crucial for detecting changes in the visual environment in the first place. With respect to AI function, involvement in saliency processing, switching of action plans, attention, and cognitive control (Menon \& Uddin, 2010) have been emphasized. Functionally, AI can be described as the general control instance the cognitive system accesses whenever actions need to be changed; thereby, actions can range from simple motor responses as in the presented study to complex forms of social interaction (White, Brislin, Meffert, Sinclair, \& Blair, 2013).

Our findings clearly emphasize the crucial role of right inferior frontal cortex and specifically rAI in action restraint and action cancellation and, thus, these specific forms of controlled behavior. It would be of interest to examine whether IFG and AI could be functionally discriminated in the context of inhibition research. This could cast further light on which specific functions-with respect to attention, updating and changing of action plans, attention, and stopping of actions-inferior frontal cortex incorporates.

\section{Right Middle and Superior Frontal Cortex}

On the basis of our fMRI localizer (see Figure 2), we expected that cTBS applied to rSFG would impair action restraint but not cancellation whereas cTBS applied to rMFG would impair action cancellation but not restraint. Our findings support the former hypothesis, although they do not support the latter.

Our findings do not suggest a crucial involvement of middle frontal cortex in response inhibition. In contrast, 
Zheng, Oka, Bokura, and Yamaguchi (2008) found a correlation between intensity of neural activation in rMFG and performance in both a GNGT and an SST and, thus, identified the middle frontal cortex as the "key locus" of inhibitory control. Neither the fMRI localizer nor the brain stimulation data in our study support this notion. Although significantly activated during action cancellation (but not action restraint), cTBS targeting rMFG did not affect the ability to inhibit in either of the tasks. In response inhibition literature middle frontal cortex is seen ambiguously: Some imaging studies find its involvement in the GNGT and the SST (e.g., Zheng et al., 2008), some don't (e.g., Rubia et al., 2001), and some find only marginal involvement (e.g., McNab et al., 2008). The only brain stimulation study including a middle frontal area as a target site (Chambers et al., 2006) did not find any effect of rTMS applied to rMFG on inhibitory processing in an SST. Our findings extend these results as we replicate the absence of an effect of GTMS applied to MFG on the SST and in addition also find no effect on the GNGT. Although the absence of evidence is no evidence of absence (De Graaf \& Sack, 2011), the described converging evidence suggests that the role of $\mathrm{rMFG}$ in response inhibition is of a less substantial but rather supportive nature. Future studies could, therefore, a priori hypothesize such a lack of evidence regarding the causal role of rMFG in response inhibition and then apply Bayesian inferential analyses to directly test zero-findings and substantiate the assumption of an expected absence of evidence as evidence of absence (see, e.g., Verbruggen, Adams, et al., 2013).

In contrast, our results might suggest the notion of a crucial involvement of superior frontal activation, specifically in action restraint. Imaging studies reported the involvement of rSFG in GNGT-related inhibition (for a review, see Simmonds et al., 2008). However, also middle frontal brain regions have been found to be activated during successful inhibition in the GNGT (for a review, see Swick et al., 2011). To our knowledge, so far neither MFG nor SFG has been investigated by means of brain stimulation in the context of action restraint (as measured in a GNGT). Our findings indicate that disruption of neural activity within rSFG might impair the ability to successfully inhibit responses in a GNGT, whereas rMFG stimulation does not. This suggests that the superior more than the middle frontal cortex is crucially involved in action restraint. Interestingly, TMS over rSFG did not affect the SST, indicating a potentially task-specific differential involvement of SFG in action restraint and not in action cancellation.

This is also in accordance with the imaging data acquired in the same sample. When directly contrasting fMRI activation during successful GNGT and SST inhibition, we found rSFG to be significantly involved in the GNGT, whereas rMFG was not. Therefore, based on our empirical fMRI data, we expected performance in the GNGT to worsen after rSFG but not rMFG stimulation. In response inhibition research, there still seems to be no consensus on which specific middle and/or superior frontal cortical structures are crucial for inhibitory processing: Simmonds et al. (2008) report a superior frontal region (BA 10) - similar to the one we find-and BA 9 in their activation likelihood estimation over 11 studies investigating the GNGT. Swick et al. (2011) report as a result of their activation likelihood estimation over 21 studies investing the GNGT a middle frontal area (BA 9) as the most crucial specific prefrontal area activated during the GNGT. Therefore, the role of middle and superior frontal areas for inhibition is still not clearly defined in fMRI literature, and this motivated our closer look into the precise distribution of specific neural activity in prefrontal areas.

In summary, our results seem to imply that superior frontal cortex is involved in action restraint as measured by a GNGT but not in action cancellation as measured by an SST, whereas middle frontal cortex does not seem to be crucial in inhibitory processing. Of course, these findings are bound to the specific stimulations sites we chose within superior and middle frontal cortex (see Table 1) based on individual functional imaging data. Furthermore, bearing in mind the rather small sample investigated in this study and the fact that effects have not been tested directly across tasks, our results regarding rSFG involvement in specific aspects of response inhibition should be interpreted with caution. In our analysis, only a priori planned comparisons (differences in false alarm rates following stimulation of certain target sites compared with the sham condition) were tested. This does not enable strong claims regarding how TMS affects each site differently, but rather interpretations on how TMS affects each site compared with a condition in which no TMs is applied. Further studies are needed to establish and test theories on superior frontal involvement in response inhibition to enable stronger claims.

\section{Pre-SMA}

Contrary to our expectations, we did not find any effect on inhibitory processing induced by disruption of neural activity within pre-SMA. In the SST participants even reacted slightly faster on go trials after pre-SMA stimulation compared with sham stimulation, which should also induce more commission errors in inhibition trials. However, pre-SMA stimulation did not elevate false alarms rates in both tasks at all. This is in opposition to a vast body of imaging literature that emphasizes consistently the involvement of pre-SMA in motor response inhibition across different inhibitory domains including the GNGT and the SST (for a review, see Swick et al., 2011). Furthermore, brain stimulation studies also reported effects of event-related single-pulse TMS (Chen et al., 2009) and tDCS (Hsu et al., 2011) applied to pre-SMA on inhibitory processing. However, it seems that these effects are difficult to replicate with repetitive TMS (see also Verbruggen et al., 2010). We explain our failure to reveal any behavioral effects on inhibitory processing for pre-SMA stimulation 
with the fact that the individual target sites for pre-SMA stimulation were located significantly deeper (mean coil cortex distance across all participants for pre-SMA $56 \mathrm{~mm}$ ) than for the other regions (mean coil cortex distance across all participants for rAI $50 \mathrm{~mm}$, for rMFG $33 \mathrm{~mm}$, for rSFG $34 \mathrm{~mm}$ ). Applying stimulation according to distance adjusted motor threshold (Stokes et al., 2007) could circumvent this problem. However, for the individual functional "hotspots" within pre-SMA we encountered, this would have exceeded common safety regulations by far (Rossi, Hallett, Rossini, \& Pascual-Leone, 2009). In their recent work, Obeso and colleagues (Obeso, Robles, Marrón, \& Redolar-Ripoll, 2013) interfered successfully with preSMA activation using online as well as offline repetitive TMS and induced inhibitory failure. Furthermore, the authors find further evidence for the essential functional alliance of pre-SMA and inferior frontal areas in response inhibition as well as for the specific involvement of preSMA in the stopping rather than the switching of actions. Therefore, we conclude that cTBS as used in the current study is likely not an appropriate method to investigate pre-SMA function. Because of methodological choices and our rather small sample size, our zero result regarding pre-SMA can only be interpreted with caution.

\section{Conclusion}

This study is the first to systematically investigate the differential involvement of several distinct functional subregions within right $\mathrm{pFC}$ in different aspects of controlled behavior. Our results emphasize the crucial role of inferior frontal cortex, specifically the rAI, in action restraint (as measured with a GNGT) and action cancellation (as measured with an SST). Furthermore, superior frontal cortex seems to be crucially involved in action restraint specifically, but not action cancellation. The role of middle frontal cortex in action inhibition seems to be of less substantial nature.

These findings enable a broader understanding of selfcontrolled behavior assigned to humans and the neural mechanism underlying inhibitory failure. In how far results acquired in the context of simple motor response inhibition can be transferred to the rather complex inhibitory processing required in everyday social interactions remains an open question for further investigations.

\section{Acknowledgments}

This work was supported by a grant from the Netherlands Organization for Scientific Research (NWO; HCMI 10-19). We thank Thomas C. Emmerling, Jeannette Boschma, and Jan Schepers for their helpful support.

Reprint requests should be sent to Franziska Dambacher, Department of Cognitive Neuroscience, Maastricht University, Oxfordlaan 55, 6229 EV Maastricht, Netherlands, or via e-mail: franziska.dambacher@maastrichtuniversity.nl.

\section{REFERENCES}

Aron, A. R., Robbins, T. W., \& Poldrack, R. A. (2004). Inhibition and the right inferior frontal cortex. Trends in Cognitive Sciences, 8, 170-177.

Band, G. P. H., Van der Molen, M. W., \& Logan, G. D. (2003). Horse-race model simulations of the stop signal procedure. Acta Psychologica, 112, 105-142.

Beeli, G., Casutt, G., Baumgartner, T., \& Jäncke, L. (2008). Modulating presence and impulsiveness by external stimulation of the brain. Behavioral and Brain Functions, 4, 33.

Boehler, C. N., Appelbaum, L. G., Krebs, R. M., Hopf, J. M., \& Woldorff, M. G. (2010). Pinning down response inhibition in the brain conjunction analyses of the stop signal task. Neuroimage, 52, 1621-1632.

Chambers, C. D., Bellgrove, M. A., Gould, I. C., English, T., Garavan, H., McNaught, E., et al. (2007). Dissociable mechanisms of cognitive control in prefrontal and premotor cortex. Journal of Neurophysiology, 98, 3638-3647.

Chambers, C. D., Bellgrove, M. A., Stokes, M. G., Henderson, T. R., Garavan, H., Robertson, I. H., et al. (2006). Executive "brake failure" following deactivation of human frontal lobe. Journal of Cognitive Neuroscience, 18, 444-455.

Chambers, C. D., Garavan, H., \& Bellgrove, M. A. (2009). Insights into the neural basis of response inhibition from cognitive and clinical neuroscience. Neuroscience and Biobehavioral Reviews, 33, 631-646.

Chen, C.-Y., Muggleton, N. G., Tzeng, O. J. L., Hung, D. L., \& Juan, C.-H. (2009). Control of prepotent responses by the superior medial frontal cortex. Neuroimage, 44, 537-545.

Chikazoe, J., Konishi, S., Asari, T., Jimura, K., \& Miyashita, Y. (2007). Activation of right inferior frontal gyrus during response inhibition across response modalities. Journal of Cognitive Neuroscience, 19, 69-80.

De Graaf, T. A., \& Sack, A. T. (2011). Null results in TMS: From absence of evidence to evidence of absence. Neuroscience and Biobehavioral Reviews, 35, 871-877.

Duann, J. R., Ide, J. S., Luo, X., \& Li, C. R. (2009). Functional connectivity delineates distinct roles of the inferior frontal cortex and presupplementary motor area in stop signal inhibition. The Journal of Neuroscience, 29, 10171-10179.

Duecker, F., \& Sack, A. T. (2013). Pre-stimulus sham TMS facilitates target detection. PloS One, 8, e57765.

Faul, F., Erdfelder, E., Lang, A.-G., \& Buchner, A. (2007). G*Power 3: A flexible statistical power analysis program for the social, behavioral, and biomedical sciences. Behavior Research Methods, 39, 175-191.

Hampshire, A., Chamberlain, S. R., Monti, M. M., Duncan, J., \& Owen, A. M. (2010). The role of the right inferior frontal gyrus: Inhibition and attentional control. Neuroimage, 50, 1313-1319.

Heatherton, T. F., \& Wagner, D. D. (2011). Cognitive neuroscience of self-regulation failure. Trends in Cognitive Sciences, 15, 132-139.

Hsu, T.-Y., Tseng, L.-Y., Yu, J.-X., Kuo, W.-J., Hung, D. L., Tzeng, O. J. L., et al. (2011). Modulating inhibitory control with direct current stimulation of the superior medial frontal cortex. Neuroimage, 56, 2249-2257.

Huang, Y.-Z., Edwards, M. J., Rounis, E., Bhatia, K. P., \& Rothwell, J. C. (2005). Theta burst stimulation of the human motor cortex. Neuron, 45, 201-206.

Jacobson, L., Javitt, D. C., \& Lavidor, M. (2011). Activation of inhibition: Diminishing impulsive behavior by direct current stimulation over the inferior frontal gyrus. Journal of Cognitive Neuroscience, 23, 3380-3387.

Logan, G. D., Schachar, R. J., \& Tannock, R. (1997). Impulsivity and inhibitory control. Psychological Science, 8, 60-64. 
McNab, F., Leroux, G., Strand, F., Thorell, L., Bergman, S., \& Klingberg, T. (2008). Common and unique components of inhibition and working memory: An fMRI, within-subjects investigation. Neuropsychologia, 46, 2668-2682.

Menon, V., \& Uddin, L. Q. (2010). Saliency, switching, attention, and control: A network model of insula function. Brain Structure and Function, 214, 655-667.

Miller, E. K. (2000). The prefrontal cortex and cognitive control. Nature Reviews, 1, 59-65.

Muggleton, N. G., Chen, C.-Y., Tzeng, O. J. L., Hung, D. L., \& Juan, C.-H. (2010). Inhibitory control and the frontal eye fields. Journal of Cognitive Neuroscience, 22, 2804-2812.

Obeso, I., Robles, N., Marrón, E. M., \& Redolar-Ripoll, D. (2013). Dissociating the role of the pre-SMA in response inhibition and switching: A combined online and offline TMS approach. Frontiers in Human Neuroscience, 7, 150.

Rossi, S., Hallett, M., Rossini, P. M., \& Pascual-Leone, A. (2009). Safety, ethical considerations, and application guidelines for the use of transcranial magnetic stimulation in clinical practice and research. Clinical Neurophysiology, 120, 2008-2039.

Rubia, K., Russell, T., Overmeyer, S., Brammer, M. J., Bullmore, E. T., Sharma, T., et al. (2001). Mapping motor inhibition: Conjunctive brain activations across different versions of go/no-go and stop tasks. Neuroimage, 13, 250-261.

Sack, A. T., Cohen Kadosh, R., Schuhmann, T., Moerel, M., Walsh, V., \& Goebel, R. (2009). Optimizing functional accuracy of TMS in cognitive studies: A comparison of methods. Journal of Cognitive Neuroscience, 21, 207-221.

Sack, A. T., \& Linden, D. E. J. (2003). Combining transcranial magnetic stimulation and functional imaging in cognitive brain research: Possibilities and limitations. Brain Research Reviews, 43, 41-56.

Sharp, D. J., Bonnelle, V., De Boissezon, X., Beckmann, C. F., James, S. G., Patel, M. C., et al. (2010). Distinct frontal systems for response inhibition, attentional capture, and error processing. Proceedings of the National Academy of Sciences, U.S.A., 107, 6106-6111.
Simmonds, D. J., Pekar, J. J., \& Mostofsky, S. H. (2008). Metaanalysis of go/no-go tasks demonstrating that fMRI activation associated with response inhibition is task-dependent. Neuropsychologia, 46, 224-232.

Stokes, M. G., Chambers, C. D., Gould, I. C., English, T., McNaught, E., McDonald, O., et al. (2007). Distance-adjusted motor threshold for transcranial magnetic stimulation. Clinical Neurophysiology, 118, 1617-1625.

Swick, D., Ashley, V., \& Turken, U. (2011). Are the neural correlates of stopping and not going identical? Quantitative meta-analysis of two response inhibition tasks. Neuroimage, 56, 1655-1665.

Varnava, A., Stokes, M. G., \& Chambers, C. D. (2011). Reliability of the "observation of movement" method for determining motor threshold using transcranial magnetic stimulation. Journal of Neuroscience Methods, 201, 327-332

Verbruggen, F., Adams, R. C., van 't Wout, F., Stevens, T., McLaren, I. P. L., \& Chambers, C. D. (2013). Are the effects of response inhibition on gambling long-lasting? PloS One, 8, e70155.

Verbruggen, F., Aron, A. R., Stevens, M. A., \& Chambers, C. D. (2010). Theta burst stimulation dissociates attention and action updating in human inferior frontal cortex. Proceedings of the National Academy of Sciences, U.S.A. 107, 13966-13971.

Verbruggen, F., Chambers, C. D., \& Logan, G. D. (2013). Fictitious inhibitory differences: How skewness and slowing distort the estimation of stopping latencies. Psychological Science, 24, 352-362.

White, S. F., Brislin, S. J., Meffert, H., Sinclair, S., \& Blair, R. J. R. (2013). Callous-unemotional traits modulate the neural response associated with punishing another individual during social exchange: A preliminary investigation. Journal of Personality Disorders, 27, 99-112.

Zheng, D., Oka, T., Bokura, H., \& Yamaguchi, S. (2008). The key locus of common response inhibition network for no-go and stop signals. Journal of Cognitive Neuroscience, 20, $1434-1442$. 